\title{
Diagnosis Experience of Patients with Cervical, Thoracic and Lumbar Multi-Segment Spinal Stenosis: A Case Report and Literature Review
}

\author{
Guosen $\mathrm{Du}^{1^{*}}$, Longbiao $\mathrm{Xu}^{{ }^{*}}$, Yajuan Tang2, Guangyu Ying2, Yongjian Zhu2\# \\ ${ }^{1}$ Department of Neurosurgery, Zhuji People' Hospital of Zhejiang Province, Zhuji Affiliated Hospital of Shaoxing University, \\ Zhuji, China \\ ${ }^{2}$ Department of Neurosurgery, Second Affiliated Hospital of Zhejiang University School of Medicine, Hangzhou, China \\ Email: "zjuzhuyj@163.com,229762580@qq.com, 18358569988@163.com, tangyajuan@zju.edu.cn, yinggy1983@163.com
}

How to cite this paper: $\mathrm{Du}, \mathrm{G} . \mathrm{S}$., $\mathrm{Xu}, \mathrm{L} . \mathrm{B}$., Tang, Y.J., Ying, G.Y. and Zhu, Y.J. (2021) Diagnosis Experience of Patients with Cervical, Thoracic and Lumbar Multi-Segment Spinal Stenosis: A Case Report and Literature Review. Case Reports in Clinical Medicine, 10, 117-125.

https://doi.org/10.4236/crcm.2021.104014

Received: March 20, 2021

Accepted: April 22, 2021

Published: April 25, 2021

Copyright $\odot 2021$ by author(s) and Scientific Research Publishing Inc. This work is licensed under the Creative Commons Attribution-NonCommercial International License (CC BY-NC 4.0). http://creativecommons.org/licenses/by-nc/4.0/ (c) (i) (9) Open Access

\begin{abstract}
Background: The incidence of cervical, thoracic and lumbar spinal canal stenosis is low. It is difficult to identify the main focus and responsible segment, and it is also difficult to select the sequence of staging surgery. We report a patient with triple stenosis. Case Presentation: In this paper, we introduced a 61-year-old female patient with cervical, thoracic and lumbar spinal canal stenosis who had previously undergone "lumbar discectomy" in the outer hospital. The postoperative effect was not good and the symptoms were poor. The diagnosis was "cervical spinal stenosis and lumbar postoperative surgery". The staged spinal canal decompression operation and Duhuo Jisheng Decoction (DHJSD) treatment were conducted in our hospital. After three months of follow-up, the functional and imaging results were satisfactory. Conclusions: The main focus and responsible spinal segment should be determined by the comprehensive analysis of medical history, signs, and images. Surgery combined with Chinese herbal medicine DHJSD therapy may be an effective treatment for this kind of disease.
\end{abstract}

\section{Keywords}

Cervical Vertebral Canal Stenosis, Thoracic Vertebral Canal Stenosis, Lumbar Vertebral Canal Stenosis, Diagnostic Strategy

\section{Background}

Spinal canal stenosis, which refers to the progressive narrowing of the spinal ${ }^{\star}$ Guosen Du and Longbiao Xu have equally contributed to this work as co-first authors. \#corresponding author. 
canal due to degeneration, is general in aged patients. The symptoms of spinal canal stenosis vary by the segment and severity of the stenosis, including radiculopathy, claudication, and myelopathy. Among all segments, the stenosis of the cervical and lumbar canal is most common due to more spinal activity [1]. Multisegmental stenosis is less common than single segmental stenosis, which can create confusion in the diagnosis and treatment. Teng et al. first reported concurrent cervical and lumbar stenosis. Dagi et al. first invent the word "tandem spinal stenosis" to describe combined stenosis, whose triad is intermittent neurogenic claudication, progressive gait disturbance, and findings of mixed myelopathy and polyradiculopathy in both the upper and lower extremities. Cervical, thoracic, and lumbar spinal canal stenosis may occur due to developmental, metabolic, or degenerative reasons. However, the diagnosis and definition of multiple spinal stenosis have not been clearly defined.

The incidence rate of multi-segment triple spinal stenosis of cervical, thoracic and lumbar vertebrae is low. At present, relevant literature reports on diagnosis and treatment are relatively rare. Park et al. analyzed the clinical data of $460 \mathrm{pa}-$ tients with lumbar stenosis, of which only 18 patients were complicated with triple stenosis. Japanese scholars found through autopsy of 1072 patients with lumbar stenosis that lumbar stenosis and thoracic stenosis are not caused by tandem stenosis, and the incidence rate of triple stenosis is relatively low [2] [3] [4]. In the following case, we presented a case of a triple spinal stenosis patient and describe our diagnosis and treatment experience in this disease.

\section{Case Presentation}

The patient, a 61-year-old woman, was admitted to the hospital for "weakness of both lower limbs for one year that aggravated with walking instability for half a year". One year ago, the patient suffered from fatigue of both lower limbs without inducement, accompanied with numbness below the plane of both knees, and could not walk for a long distance, while both upper limbs were not affected. No palpitation, chest tightness, dizziness, nausea and other discomforts. In the following six months, the symptoms gradually worsened that the patient had the feeling of stepping on cotton and walked unstably. The patient was diagnosed with "lumbar disc herniation and lumbar spinal canal stenosis" in the outer hospital and underwent lumbar discectomy (Figure 1(a) and Figure 1(b)). The above symptoms did not ease after operation. In the past six months, the patients felt that the appeal symptoms were further aggravated and accompanied by walking difficulties. Therefore, she turned to our hospital for further treatment and was admitted to our department as "cervical spinal stenosis and lumbar postoperative surgery".

The patient had the past history of "left knee arthroplasty" and "left femoral internal fixation" for the left femoral fracture (Figure 1(c)).

As to the physical examination, positive Hoffmann sign was found of both upper limbs, and muscle strength of the right lower limb was Grade IV/V, III/V of the left lower limb; muscle tension of bilateral limbs was normal, with bilater- 
al superficial hypoesthesia below knee joint; and bilateral ankle clonus and Babinski signs were positive.

Imaging examination: Cervical magnetic resonance (June 18th, 2019) discovered intervertebral disc herniation of the cervical 3 - 7 segments, ligamentum flavum thickening and calcification of cervical $4 / 5$ segment, cervical canal stenosis, and cervical spinal cord compression (Figure 2(a) and Figure 2(b)); Thoracic vertebral magnetic resonance (June 23rd, 2019) revealed the intervertebral disc herniation of thoracic 10/11 and 11/12 segments, hypertrophy and ossification of ligamentum flavum, and corresponding spinal canal stenosis; Spinal cord edema at thoracic 10 to 11 segments (Figure 2(c)).

Based on the history, positive vertebral bundle sign and weakness of both lower limbs and imaging findings, we hold that the cervical spinal stenosis was responsible for the symptoms therefore decompression of the cervical spinal tube could be helpful to relieve the symptoms. Meanwhile, the patient was reluctant to more operations due to her unpleasant previous experience. We, therefore, planned to perform cervical spinal decompression as the one-stage treatment. Improvement was expected as a support for patient's confidence for further decompression of thoracic spinal stenosis.

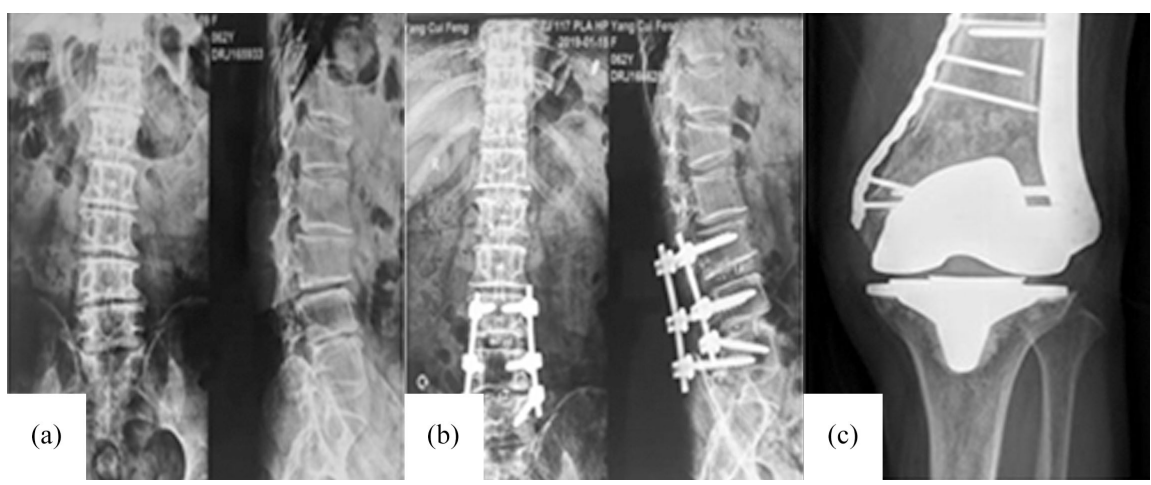

Figure 1. Preoperative (a) and postoperative (b) for lumbar disc herniation fusion and internal fixation; Left knee arthroplasty and femoral internal fixation (c).

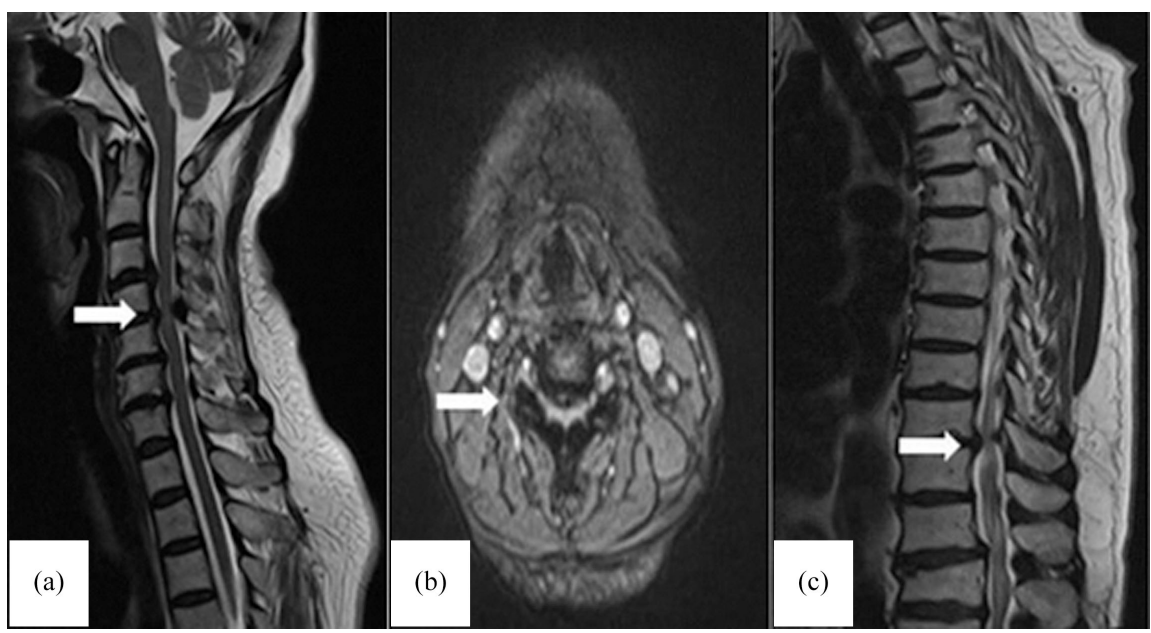

Figure 2. Cervical 4/5 spinal canal stenosis (a and b); thoracic 10 spinal canal stenosis (c). 
The patient first underwent cervical 4/5 spinal canal decompression and partial resection of ligamentum flavum under general anesthesia, and went through the postoperative stage smoothly. The patients were treated with anti-infection and nerve nutrition after operation. ECG monitoring, oxygen inhalation, keeping respiratory tract unobstructed, lying position rest, preventing bedsore, pulmonary infection, urinary tract infection, and deep vein thrombosis, guiding functional exercise were conducted. She was satisfied with the postoperative improvement, and the numbness of both lower limbs was obviously relieved than before. The muscle strength of the right lower limb was Grade IV/V, while strength of the left lower limb remained the same. After two weeks, the thoracic 10/11 spinal canal decompression was followed, and the same postoperative management was developed. The numbness of both lower limbs was significantly relieved. The muscle strength of the right lower limb was $\mathrm{IV}^{+} / \mathrm{V}$, improved compared with that before operation. The left lower limb was still felt heavy but the sense of movement was improved. During hospitalization and after discharge, this patient was also treated with DHJSD, with the dosage of $100 \mathrm{ml}$ per day for three months.

With the three months follow-up after operation, the patient's residual symptoms improved significantly, and the functional and imaging effects were satisfied (Figure 3). The standing balance was more stable than before, and the patient could walk a longer distance with the aid of auxiliary devices. The muscle tension of both upper limbs was normal, Hoffmann sign was negative, muscle strength was Grade V/V, normal tendon reflex. Muscle tension of both lower limbs improved, with bilateral iliopsoas muscle strength $\mathrm{Grade}_{\mathrm{IV}} / \mathrm{V}$, quadriceps femoris muscle strength Grade IV/V, tibialis anterior muscle and gastrocnemius muscle strength Grade V/V. Bilateral patellar clonus was negative and bilateral ankle clonus was positive. The superficial sensation below the bilateral knee joint was improved with intact positional sensation, and Babinski sign was not observed.

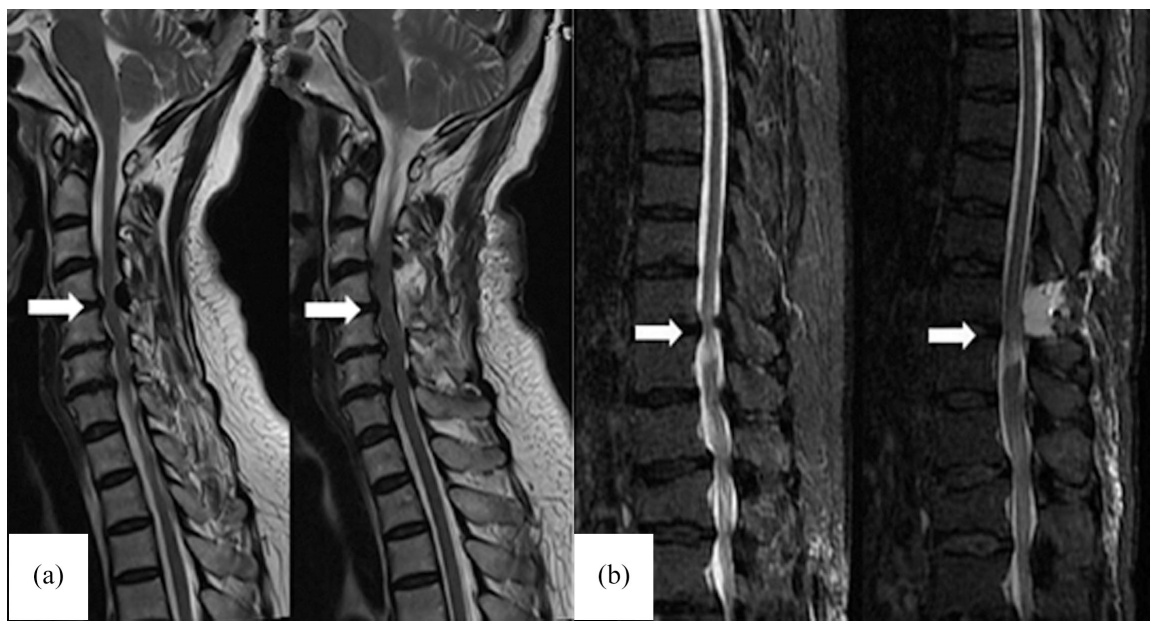

Figure 3. Preoperative and postoperative for cervical 4/5 spinal canal stenosis (a); preoperative and postoperative for thoracic 10 spinal canal stenosis (b). 


\section{Discussion}

Most patients with cervical, thoracic and lumbar multi-segment spinal stenosis have a long course of the disease and often have experienced various treatment experiences. It is difficult to verify the development and changes of the diseases. The patient in our case had undergone two previous surgical treatments for her symptoms, and neither of them had achieved expected results nor gotten worse. The decision-making process of diagnosis and treatment is worthy of our consideration.

Such patients mostly have both upper and lower motor neuron symptoms, and the symptoms are interwoven with each other, which is easy to be missed and misdiagnosed [5]. In the process of diagnosis and treatment, the medical history should be inquired in detail to guide patients to provide valuable information for diagnosis. It is particularly important to carry out a careful physical examination, which can identify the main focus and responsible segment by paying attention to the unique positive signs of cervical, lumbar and thoracic vertebral diseases and making analysis and judgment. In addition, imaging exams are also helpful for evaluation, like X-rays of hyperextension and hyperflexion, CT scan with three-dimensional reconstruction, MRI of relevant brain and spine, nerve electrophysiology examination, etc. Therefore, complete history taking, careful physical exams for positive signs, as well as imaging findings are the three elements for an exact diagnosis. Only imaging changes without definite clinic manifestation and/or positive physical signs are not enough for the treatment plan making especially when the patient has multiple segmental problems [6] [7].

The sequence of staging surgery is also tough in this case. This patient suffered from walking instability and weakness, foot stepping on cotton sensation, and intermittent claudication of spinal cord origin. The patients were found with increased bilateral muscle tension and more activated tendon reflex, and positive Babinski sign, Hoffmann sign, and ankle clonus, those were the typical symptoms of cervical spinal cord upper motor neuron compression. Therefore we figured out that the cervical spinal canal stenosis was the responsible segment and should be treated predominantly. Currently, priority is given to segments, $\mathrm{Wu}$ et al. gave priority to cervical spine surgery for 222 patients with cervical spine complicated with lumbar spinal stenosis. Comparing the postoperative JOA score with Nurick score, it is concluded that the first cervical spine surgery can significantly improve the postoperative JOA score and ODI index and reduce the second-stage operation rate, [8] and Luo et al. pointed out that if lumbar spinal stenosis was treated first in patients with the cervical spine as the main focus, the symptoms related to cervical spinal stenosis would deteriorate rapidly [9] [10]. Therefore, for patients with "cervical, thoracic and lumbar triple stenosis", it is very necessary to perform the whole spinal MRI examination before spinal canal decompression surgery, especially before decompression surgery for patients with lumbar spinal tube stenosis when positive findings cannot be rea- 
sonably explained by pure lumbar lesions. So as to exclude the possibility of multi-segment stenosis and other lesions such as mass lesions or vascular lesions [11]. In our country, whole spine examination is sometimes limited by financial elements, and patients are hardly followed by the same doctor in the outpatient. These make it difficult for an exact diagnosis and probable treatment. This phenomenon is also an important factor that causes clinical misdiagnosis.

At present, Surgical treatment plays an important role in treating spinal stenosis, which can reduce the clinical symptoms, while there are problems such as infection, complications, and high postoperative recurrence rate [12] [13] [14]. Except for surgical treatment, nonsurgical therapy, including traction, manipulation, acupuncture, physiotherapy, and medicines, especially Chinese herbal medicine showed good effects [15] [16] [17] [18]. Chinese herbal medicine has been used in clinical practice for a long time and Duhuo Jisheng Decoction (DHJSD) is one of the representative prescriptions [19] [20]. The ancient classic prescriptions represented by DHJSD were recorded in the book "Bei Ji Qian Jin Yao fang" written by Sun Simiao of the Tang Dynasty. It consists of 15 commonly used Chinese herbs. According to the theory of TCM, it has the effects of eliminating rheumatism, analgesics, nourishing liver, and kidney, nourishing Qi and blood, and channeling meridians [21] [22]. Basic research shows that it has the effects of anti-inflammatory, analgesia, immune regulation, cartilage promotion, and fibrous ring repair [23]. DHJSD has been widely used in the treatment of lumbar disc herniation [24] [25], and other orthopedic diseases, such as knee arthritis and osteoporosis [26] [27]. A systematic review and meta-analysis for DHJSD in the treatment of lumbar disc herniation also showed good effectiveness and safety [23]. In our case, after surgery, this patient was treated with DHJSD, with the dosage of $100 \mathrm{ml}$ per day for three months. With the follow-up, the patient's symptoms improved significantly. It is proved that surgery combined with Chinese herbal medicine DHJSD therapy is an effective treatment for this patient.

\section{Conclusion}

We should pay more attention to patients with spinal degenerative diseases, and must pursue the unity of medical history, signs, and images. In case of difficult patients, more comprehensive examinations are required, and the main focus and responsible segment are determined through comprehensive analysis. Further exploration is needed when the manifestation could not be reasonably explained by the acquired information, especially when some focal lesions exist while some potential coexisted problems are ignored due to incomplete examinations.

\section{Ethics Approval and Consent to Participate}

This study was approved by the clinical ethics committee of the Second Affiliated Hospital of Zhejiang University School of Medicine. The patient had been 
anonymized and the informed consent was acquired.

\section{Availability of Data and Materials}

Data sharing not applicable to this article as no datasets were generated or analysed during the current study.

\section{Funding}

This work was supported by Projects of Shaoxing Science and Technology Bureau (No. 2018C30150) and Zhejiang Medical and Health Science and Technology (No. 2020PY081).

\section{Authors' Contributions}

This case report was designed by XL and ZY, case materials were collected by TY, this article was written by XL and TY. YG and ZY participated in discussion development, reviewed the article, and provided expert guidance. All authors read and approved the final manuscript.

\section{Conflicts of Interest}

The authors declare no conflicts of interest regarding the publication of this paper.

\section{References}

[1] Li, W., Guo, S., Sun, Z., et al. (2015) Multilevel Thoracic Ossification of Ligamentum Flavum Coexisted with/without Lumbar Spinal Stenosis: Staged Surgical Strategy and Clinical Outcomes. BMC Musculoskeletal Disorders, 16, Article No. 206. https://doi.org/10.1186/s12891-015-0672-5

[2] Park, M.S., Moon, S.H., Kim, T.H., et al. (2015) Asymptomatic Stenosis in the Cervical and Thoracic Spines of Patients with Symptomatic Lumbar Stenosis. Global Spine Journal, 5, 366-371. https://doi.org/10.1055/s-0035-1549031

[3] Bajwa, N.S., Toy, J.O. and Ahn, N.U. (2012) Is Lumbar Stenosis Associated with Thoracic Stenosis? A Study of 1,072 Human Cadaveric Specimens. The Spine Journal, 12, 1142-1146. https://doi.org/10.1016/j.spinee.2012.10.029

[4] Kim, B.S., Kim, J., Koh, H.S., et al. (2010) Asymptomatic Cervical or Thoracic Lesions in Elderly Patients Who Have Undergone Decompressive Lumbar Surgery for Stenosis. Asian Spine Journal, 4, 65-70. https://doi.org/10.4184/asj.2010.4.2.65

[5] Yamada, T., Yoshii, T., Yamamoto, N., et al. (2018) Surgical Outcomes for Lumbar Spinal Canal Stenosis with Coexisting Cervical Stenosis (Tandem Spinal Stenosis): A Retrospective Analysis of 565 Cases. Journal of Orthopaedic Surgery and Research, 13, Article No. 60. https://doi.org/10.1186/s13018-018-0765-6

[6] Naderi, S. and Mertol, T. (2002) Simultaneous Cervical and Lumbar Surgery for Combined Symptomatic Cervical and Lumbar Spinal Stenoses. Journal of Spinal Disorders \& Techniques, 15, 229-232. https://doi.org/10.1097/00024720-200206000-00011

[7] Patel, E.A. and Perloff, M.D. (2018) Radicular Pain Syndromes: Cervical, Lumbar, and Spinal Stenosis. Seminars in Neurology, 38, 634-639.

https://doi.org/10.1055/s-0038-1673680 
[8] Li, H., Chen, Z., Li, X., et al. (2017) Prioritized Cervical or Lumbar Surgery for Coexisting Cervical and Lumbar Stenosis: Prognostic Analysis of 222 Case. International Journal of Surgery, 44, 344-349. https://doi.org/10.1016/j.ijsu.2017.07.027

[9] Luo, C.A., Kaliya-Perumal, A.K., Lu, M.L., et al. (2019)Staged Surgery for Tandem Cervical and Lumbar Spinal Stenosis: Which Should Be Treated First? European Spine Journal, 28, 61-68. https://doi.org/10.1007/s00586-018-5795-6

[10] Matsumoto, M., Okada, E., Ichihara, D., et al. (2010) Age-Related Changes of Thoracic and Cervical Intervertebral Discs in Asymptomatic Subjects. Spine, 35, 1359-1364. https://doi.org/10.1097/BRS.0b013e3181c17067

[11] Fushimi, K., Miyamoto, K., Hioki, A., et al. (2013) Neurological Deterioration Due to Missed Thoracic Spinal Stenosis after Decompressive Lumbar Surgery: A Report of Six Cases of Tandem Thoracic and Lumbar Spinal Stenosis. The Bone \& Joint Journal, 95, 1388-1391. https://doi.org/10.1302/0301-620X.95B10.31222

[12] Dave, B.R., Degulmadi, D., Krishnan, A., et al. (2019) Risk Factors and Surgical Treatment for Recurrent Lumbar Disc Prolapse: A Review of the Literature. Asian Spine Journal, 10, 1-9.

[13] Chen, X.L., Uphar, C., Samuel, L., et al. (2019) Complication Rates of Different Discectomy Techniques for the Treatment of Lumbar Disc Herniation: A Network Meta-Analysis. European Spine Journal, 28, 2588-2601. https://doi.org/10.1007/s00586-019-06142-7

[14] Li, Z., Hao, Y., Liu, M., et al. (2018) Clinical Characteristics and Risk Factors of Recurrent Lumbar Disk Herniation: A Retrospective Analysis of Three Hundred and Twenty-One Cases. Spine, 43, 1463-1469. https://doi.org/10.1097/BRS.0000000000002655

[15] Tang, S.J., Mo, Z.M. and Zhang, R.W. (2018) Acupuncture for Lumbar Disc Herniation: A Systematic Review and Meta-Analysis. Acupuncture in Medicine, 36, 62-70. https://doi.org/10.1136/acupmed-2016-011332

[16] Mo, Z.M., Zhang, R.W., Chen, J.F., et al. (2018) Comparison between Oblique Pulling Spinal Manipulation and Other Treatments for Lumbar Disc Herniation: A Systematic Review and Meta-Analysis. Journal of Manipulative and Physiological Therapeutics, 41, 771-779. https://doi.org/10.1016/j.jmpt.2018.04.005

[17] Zhang, W., Guo, W., Zhao, P., et al. (2013) Therapeutic Effects of Chinese Osteopathy in Patients with Lumbar Disc Herniation. The American Journal of Chinese Medicine, 41, 983-994. https://doi.org/10.1142/S0192415X13500663

[18] Zhi, X.C., Xu, J. and Qian, X.Z. (2018) Advances in the Conservative Treatment of Lumbar Intervertebral Disc Herniation. China Medical Herald, 15, 36-39.

[19] Lin, X.J. and Chen, C.Y. (2007) Advances on Study of Treatment of Lumbar Disk Herniation by Chinese Medicinal Herbs. China Journal of Chinese Materia Medica, 32, 186-191.

[20] Chen, X.h., Fan, Y.h. and Yu, Z. (2017) Clinical Research Progress on Herbal Oral Administration Forlumbar Disc Herniation. Tianjin University of Traditional Chinese Medicine, 36, 237-240.

[21] Pan, C.B., Feng, Y. and Liu, X.X. (2013) Discussion on the Connotation and Extension of Duhuo Jisheng Decoction. Arthritis \& Rheumatology, 2, 39-41.

[22] Lin, Y.M. and Qiu, J. (2019) Research Progress of Duhuo Jisheng Decoction in Treating Lumbar Disc Herniation. Yunnan Journal of Traditional Chinese Medicine and Materia Medica, 40, 79-81.

[23] Sun, K., Huang, F., Qi, B., Yin, H., et al. (2020) A Systematic Review and Meta-Analysis for Chinese Herbal Medicine Duhuo Jisheng Decoction in Treatment of 
Lumbar Disc Herniation. A Protocol for a Systematic Review. Medicine, 99, e19310. https://doi.org/10.1097/MD.0000000000019310

[24] Hao, Y.Y. (2017) The Curative Effect and Mechanism of Duhuo Jisheng Decoction on Lumbar Intervertebral Disc Protrusion of Liver-Kidney Deficiency Type. Clinical Research and Practice, 2, 119-120.

[25] Xu, M. and Jiang, H. (2018) The Clinical Efficacy and Prognosis of Duhuo Jisheng Decoction in the Treatment of Cold Dampness Type Lumbar Intervertebral Disc Herniation. Shaanxi Zhongyi, 39, 157-159.

[26] Liu, S.Z. and Wang, J.T. (2018) Clinical Observation of Duhuo Shengtang Decoction in Treating Postmenopausal Women with Osteoporosis. China Journal of Chinese Materia Medica, 34, 181-183.

[27] Zhang, W.M., Wang, S.Q., Zhang, R.X., et al. (2016) Evidence of Chinese Herbal Medicine Duhuo Jisheng Decoction for Knee Osteoarthritis: A Systematic Review of Randomised Clinical Trials. BMJ Open, 6, e008973.

https://doi.org/10.1136/bmjopen-2015-008973

\section{List of Abbreviations}

Computed tomography (CT); Duhuo Jisheng Decoction (DHJSD); magnetic resonance imaging (MRI); Japanese Orthopedics Associati (JOA); Oswestry Disability Index (ODI). 